\title{
Chromatographic Fingerprinting Coupled with Chemometrics for Quality Control of Traditional Chinese Medicines
}

\author{
Yi-zeng Liang ${ }^{\star a}$ and Wei-Ping Wang ${ }^{\text {ab }}$
}

\begin{abstract}
The holistic system of traditional Chinese medicine (TCM) is reflected by the integrity of the ingredients contained in herbal medicines, which creates a challenge in establishing quality control standards for raw materials and the standardization of finished herbal drugs because no single component contributes to the total efficacy. Thus, the chromatographic fingerprinting technique of TCM has proved to be a comprehensive strategy for assessing the intact quality of herbal medicine, since the origin of the herbal medicines could be identified and classified based on so-called phytoequivalence. On the other hand, chromatographic fingerprinting is essentially a high-throughput technique and an integral tool to explore the complexity of herbal medicines. In order to further control the comprehensive quality of TCMs, some strategies are proposed to trace the chemical changes of chromatographic fingerprints both in product processing and/or after their administration by modern chromatographic techniques and chemometrics. Combined with the techniques developed in systems biology, it seems also possible to reveal the working mechanism of TCMs and to further control their intrinsic quality.
\end{abstract}

Keywords: Chemometrics · Chromatographic fingerprinting · Traditional Chinese medicine

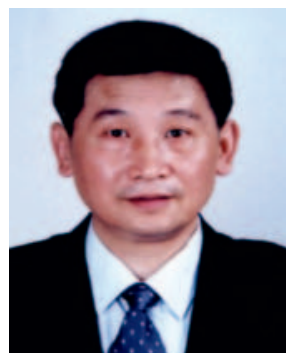

Yi-zeng Liang obtained his Ph.D. in analytical chemistry at Hunan University in1988. In 1994, he earned the Dr. philos. at University of Bergen, Norway. He is a professor of chemometrics and analytical chemistry in Central South University (CSU). He leads the research centre of modernization of traditional chinese medicines in CSU. He is vice chairman of the Computer Chemistry Committee, the

${ }^{\star}$ Correspondence: Prof. Dr. Y.-z. Liang ${ }^{a}$

${ }^{a}$ Research Center of Modernization of Chinese Medicines

Central South University

Changsha 410083, China

E-mail: yizeng_liang@263.net

bXinjiang Uygur Autonomous Region Institute for Food and Drug Control

Urumqi, 830004, China
Chemical Society of China (since 2001), editor of Chemometrics and Intelligent Laboratory Systems (since 2007). His research interests include analytical chemometrics and bioinformatics, chemical fingerprinting and quality control of traditional Chinese medicines; data mining in chemistry, metabolomics etc. Professor Liang has published more than 360 scientific research papers in the source journals of SCI since 1989. The SCI citation number is more than 5300 with h-index of 32 . He has published 10 books ( 8 in Chinese and 2 in English) and 6 chapters (chapter author) in 3 books in English.

\section{Introduction}

Western and traditional Chinese medical practices represent completely different philosophies.[1] In short, traditional Chinese medicine (TCM) focuses on the diseased person instead of the person's disease, which is the main concern of typical western medicine. ${ }^{[2]}$ TCMs usually contain multiple botanicals, each of which contains many compounds that may be relevant to the medicine's putative activity. However, research on the efficacy and quality control of TCMs is far from the standards of modern science. Thus, it is a big challenge for us to find a way to effectively control the quality of TCMs and to efficiently explore the working mechanisms of TCMs. [3] These are both the theoretical and practical questions to be answered by research on the modernization of TCM.

A western scientist, analyzing western and Chinese medicine, reflects that "the two approaches boil down to a simple question: is it better to attack disease as the western world does, with a silver bullet - the one substance whose potency has been pinpointed? Or should treatment be administered, as the Chinese method dictates, by aiming a group of agents at the problem - the shotgun approach"? In fact, which one is better depends on the viewpoint. In general, from the point of view of science, the western medical system, especially at the theoretical level, has a much stricter and more comprehensive basis, compared with traditional medicine, which is mainly based on long experience. However, a number of studies have supported the efficacy and safety of some TCM herbal formulas. For instance, a randomized, non-blind, controlled clinical trial conducted in Japan showed that sho-saiko-to, an extract of seven Chinese herbs, helps prevent liver cancer in patients with cirrhosis. ${ }^{[4]}$ Also, two doubleblind, placebo-controlled clinical trials were performed in Britain to evaluate the effect of Zemaphyte, a preparation of ten herbs used by TCM practitioners for treating certain kinds of skin disease. In both studies, the formula produced impressive responses in treating severe, widespread atopic eczema that was resistant to conventional steroid therapies. ${ }^{[5,6]}$ Thus, some western-trained scientists have long expressed the view that the benefits of TCM 
drugs often come as a result of synergistic interactions of multiple ingredients. ${ }^{[7-9]} \mathrm{It}$ follows that the reductionist approach of isolation of a single bioactive compound is not always appropriate for TCM. More recently, Simon Frantz, a news editor for Nature Reviews Drug Discovery, said, "Forget drugs carefully designed to hit one particular molecule - a better way of treating complex diseases such as cancer may be to aim for several targets at once". ${ }^{[10]}$ The reason for this is that common disorders such as cancer, cardiovascular disease, and depression tend to result from multiple molecular abnormalities, not from a single defect. What's more, pinpointing a single target is unlikely to help in many cases because cells can often find ways to compensate for a protein whose activity is affected by a drug, a phenomenon known as redundancy. Using so-called 'magic shotguns' to target multiple points in these complex systems, could reap bigger therapeutic rewards than fully blocking one target. The idea of the magic bullet continues to be a great idea, but in practice it's probably not going to be the right approach for complex diseases. ${ }^{[10]}$ A number of companies and research groups are now screening compounds that stick to several targets, or are even trying to engineer promiscuous drugs. Arguably, the biggest area for promiscuous drugs at the moment is cancer. ${ }^{[11]}$ Furthermore, Kitano, a Japanese scientist, even proposed systems-oriented drug design. He pointed out that Chinese medicine can be an inspiration to create a drug discovery strategy that uses large numbers of components to systematically control a complex network system. ${ }^{[12]}$ Given that robustness is a fundamental property of biological systems, systems-based approaches to future drug design should consider robustness as the central framework. He said, "a new way of controlling complex and robust biological systems through higher-complexity drugs will need to be established, as complexity has to be controlled by complexity, but addressing these challenges and making robustness based approaches to drug design a reality could cause a fundamental transformation in the drug industry and in medical practice".

Anyway, regardless of the type of medical system, quality-consistent medication is the prerequisite for efficacy assurance. Referring to the above mentioned, let us take the symbolized 'silver bullet' or 'shotgun' description to discuss how to properly assess the quality of Chinese herbal medicines. The conventional purpose is to identify false from true, test for impurities and determine the content of the selected single chemical entity by appropriate analytical technologies. But in recent years, quality judgment by determining one or two markers and ignoring the complexity of the herbal medications is no longer the central consideration, and there is now consensus that a comprehensive strategy for assessing the intact quality of herbal medicine is necessary. Consequently, chromatographic fingerprinting $(\mathrm{CF})$ and simultaneous determination of multiple components are the current trends. ${ }^{[13-15]}$ Moreover, in order to reveal the working mechanisms of TCMs and to further explore the features in the chromatographic profile responsible for bioactivity, it is necessary for techniques developed recently in systems biology, such as metabolomics and proteomics to be introduced into the research field of modernization of TCMs. Of course, the techniques developed in both chemometrics and bioinformatics dealing with the complex data from TCMs and biological measurements will also be necessary for this purpose.

\section{Quality Control of TCMs based on Chromatographic Fingerprinting and Chemical Pattern Recognition}

The quality control of TCMs has been a challenge in TCM research because of their great variety and the most complex pharmacokinetics. Many factors, such as time of harvest, weather conditions, locations, quality of soil and genetic factor, will influence the quality of the herbal medicines. At the same time, techniques used for treating and processing Chinese herbal medicines, such as drying, cutting, extracting etc., have also a great influence upon the quality of the intermediate products and final products of TCMs. By definition, chromatographic fingerprinting of TCMs is a comprehensive, quantifiable tool for phytochemical identification with its basic attributes of 'integrity' and 'fuzziness'.[16-18] Thus, with the help of multivariate pattern analysis methods developed in chemometrics, chromatographic fingerprinting can be used as a technique for the authentication of species and evaluation of the stability and consistency of the raw materials, semiproducts and final products of TCMs. ${ }^{[19-35]}$

Let us consider a concrete example. Epimedium herb (Yin yang huo in Chinese), a popular Chinese herb, derives from a multiple species colony belonging to the family Berberidaceae. Most of the species are distributed in various provinces in China. There are five species of Epimedium adopted in the Chinese pharmacopoeia: $E$. brevicornu Maxim., E. koreanum Nakai, E. sagittatum (Sieb. et Zucc.) Maxim., E. pubescens Maxim., and E. wushanense T. $S$. Ying. A survey of resources indicated that Epimedium pubescens, E. brevicornum, and $E$. koreanum are in the commercial mainstream of the CMM market. Beyond the five official species, E. acuminatum, E. leptorrhizum and others are also distributed in local markets and could be mixed in with the first ones. Official Epimedium species have commonly C-8prenylated flavonol glycosides, which show an immunomodulatory effect, osteoblastic proliferative activity, and sex hormone activities as reported elsewhere. Therefore icariin, epimedin A, epimedin $\mathrm{B}$, and epimedin $\mathrm{C}$ are unanimously used as bioactive marker compounds for quality control. Hence, selecting the highcontent prenylated flavonoids as specific ingredients in the Epimedium herb to identify species and assess the inherent quality is a rational choice when carrying out chromatographic fingerprint analysis. The HPLC profiles of the extracts of leaves of the five official Epimedium species, namely $E$. koreanum, E. brevicornu, E. pubescence, E. wushenense, and E. sagigattum showed a common specific region between retention times of about 37 to $50 \mathrm{~min}$, which consisted of 5 to 7 peaks involving the most important four prenylated flavonoids, i.e. epimedin A (peak 2), epimedin B (peak 3), epimedin C (peak 4), and icariin (peak 6) as well as 1 to 3 minor unknown flavonoids peaks (see Fig. 1). The above-mentioned fingerprint region of HPLC profiles of Epimedium spp. represents the specificity for authentication and quality assessment. Different ratios between the peaks in this region constitute the independent characteristics of the five official species. Briefly, the dominant epimedin $\mathrm{C}$ peak (peak 4) in the region is characteristic of E. wushanense (E.w. pattern), icariin (peak 6) is the strongest peak found in $E$. koeanum (E.k. pattern) extract, epimedin B (peak 3) together with icariin (peak 6) is the defining feature of E. brevicornum (E.b. pattern), and E. pubescens (E.p. pattern) and E. sagittatum (E.s. pattern) are identified by their prevailing peaks of epimedin C (peak 4) and icariin (peak 6) in the region whereas peak 4 is generally higher than peak 6 in the 'E. s. pattern' and peak 6 is often stronger than peak 4 in the 'E. p. pattern', respectively. Using principle component analysis (PCA), analysis of 46 samples of Epimedium species, and taking the HPLC profile of $E$. koreanum as a reference, five patterns were categorized clearly. The first principle component (PC1) is epimedin C (score 0.68 ), and the second (PC2) is epimedin $\mathrm{B}$ and icariin (scores 0.70 and 0.69, respectively), this indicates that the most influential factors for Epimedium spp. are concentrated in this region. It appears that in spite of the E.b. pattern, the epimedin C (peak 4) declines from dominance in the E.w. pattern in the order of E.w. pattern 


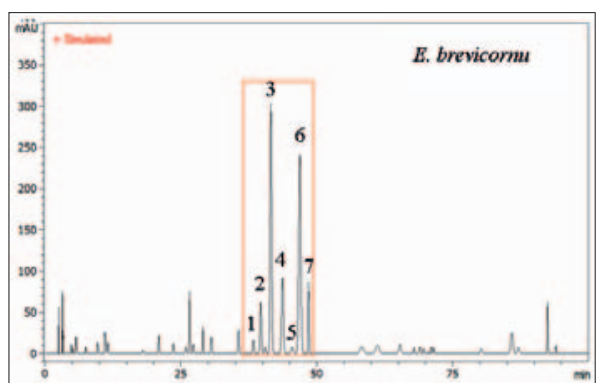

Fig. 1. HPLC profiles of the leaves of the five official Epimedium species, namely $E$. brevicornu, E. koreanum, E. wushenense, $E$. pubescence, E. sagigattum (from top left to bottom right). In the specific region between retention times 37-50 min (marked by the frames), there are 5-7 peaks involving the most important four prenylated flavonoids, epimedin A (peak 2), epimedin B (peak 3), epimedin C (peak 4), and icariin (peak 6), respectively.

$\leftarrow$ E.s. pattern $\leftarrow$ E.p. pattern $\leftarrow$ E.k. pattern (see Fig. 2 for details). Previous papers published on HPLC fingerprinting analysis ${ }^{[36-39]}$ of the Epimedium species focused on this region as the characteristic HPLC profile of Epimedium herbs for phytotaxonomy and quality analysis. For example, Guo and coworkers ${ }^{[37]}$ attempted to delineate the subtle variation of this region of 35 species of Epimedium to differentiate the species more strictly. All the samples tested were divided into four main types and nine subtypes to comply with the complex morphological taxonomic system devised by W.T. Stearn. ${ }^{[40,41]}$ Plant taxonomy needs a divergence strategy to distinguish the visual variance of the appearance in a subtle way to define the uniqueness of species. The complex classification verified the diversity of the botanical kingdom. However, to solve the practical difficulties caused by multiple species coexisting on the CMM market as herbal medicine commodities, the
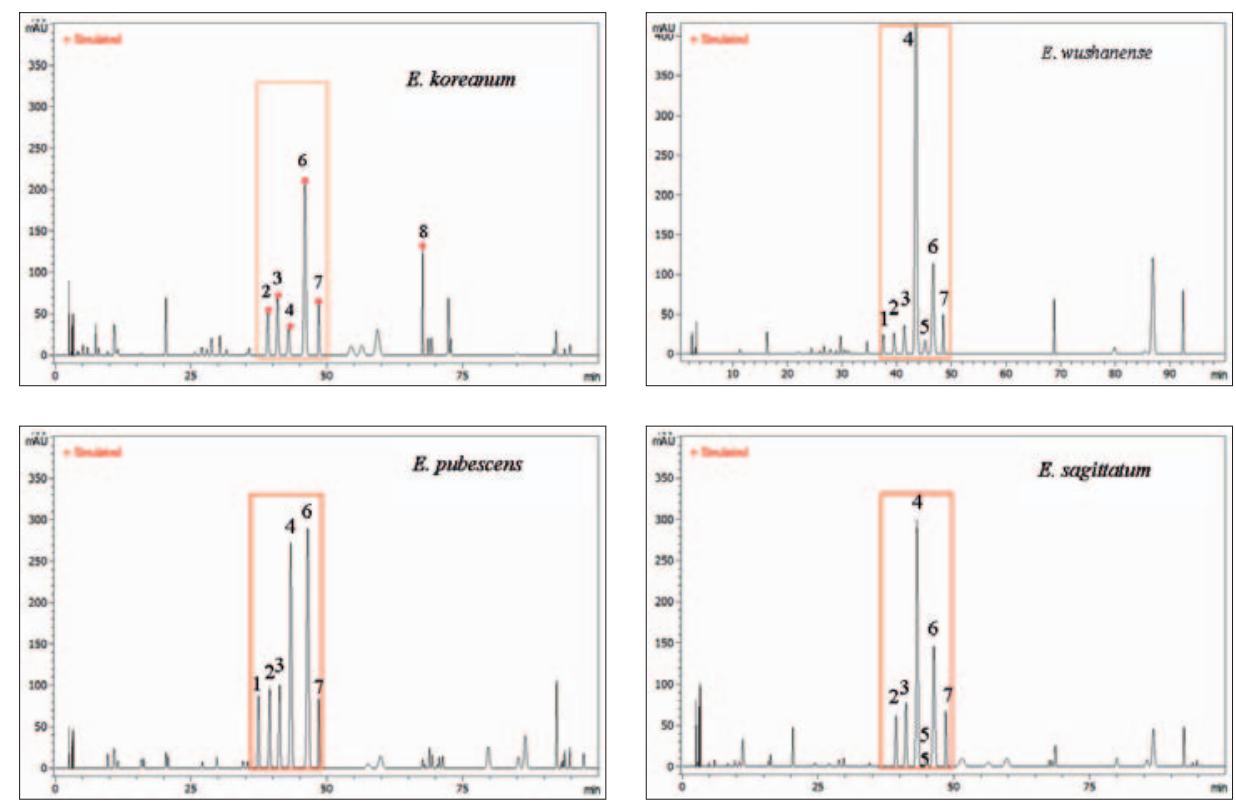

divergence approach is obviously not the right choice. To converge the different species that possess the same HPLC fingerprint pattern towards one group will facilitate the efficacious use of multiple species of CMM such as the Epimedium species. Convergence or divergence obviously depends on the final purpose. ${ }^{[42]}$

In fact, the chromatographic fingerprints of Chinese herbal medicines focus mainly on the secondary metabolites of the plants. Thus, even the influence of cultivation of the plants, including time of harvest, weather conditions, location, quality of soil, can also be described. Fig. 3 shows such an example. GC-MS fingerprints of 24 volatile constituents of Pericarpium Citri Reticulatae and Pericarpium Citri Reticulatae Viride collected at different harvesting periods are shown in Fig. 3(A). They are quite similar but with some minor differences. Is there some relationship among them? The result of pattern analysis based on principal component analysis

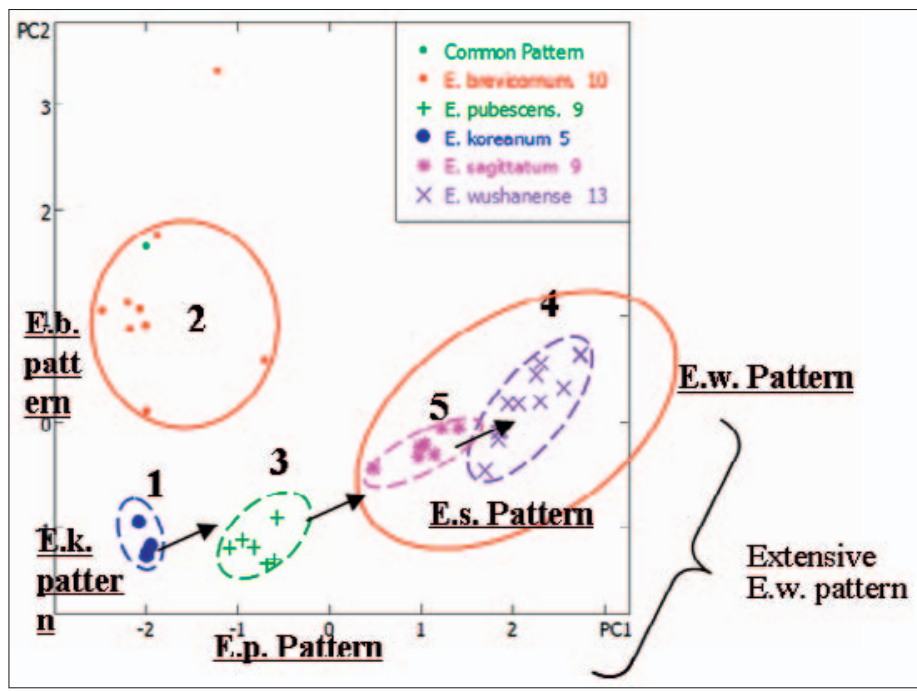

Fig. 2. The score plot of principle component analysis of 46 samples of Epimedium spp.
(PCA) answers the question. Fig. 3(B) shows the result, which looks like their footprint at different growing periods.

Furthermore, a compound medicine of Dangguibuxuetang and its two involved single medicines of Danggui
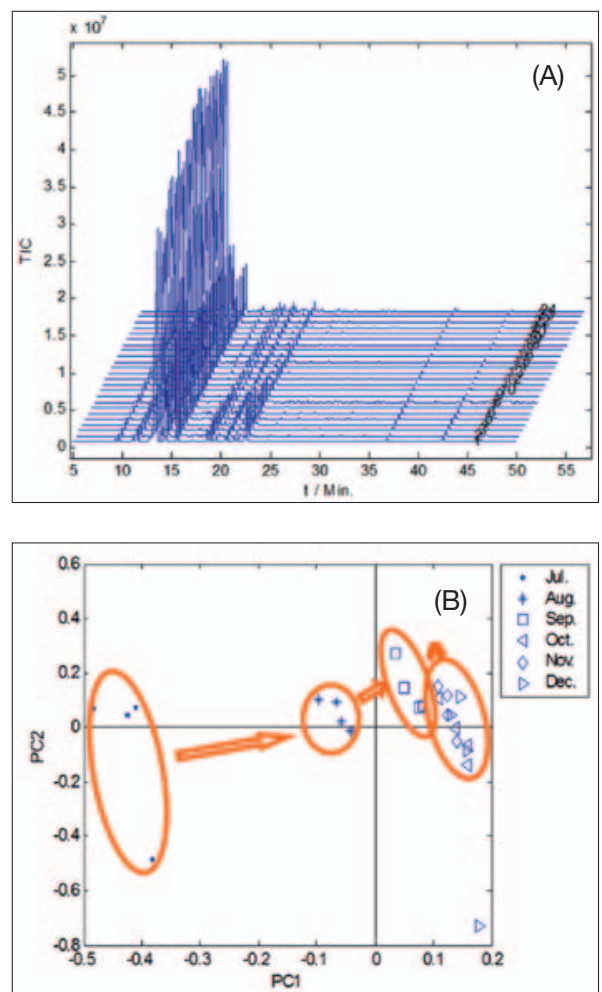

Fig. 3. Illustration of the influence of harvest time on Chinese herbal medicines through chromatographic fingerprints. (A) 24 chromatographic fingerprints of volatile constituents of Pericarpium Citri Reticulatae and Pericarpium Citri Reticulatae Viride collected at different harvesting periods obtained by GC-MS. (B) Analysis results of the 24 chromatographic fingerprints of volatile constituents of Pericarpium Citri Reticulatae and Pericarpium Citri Reticulatae Viride. 


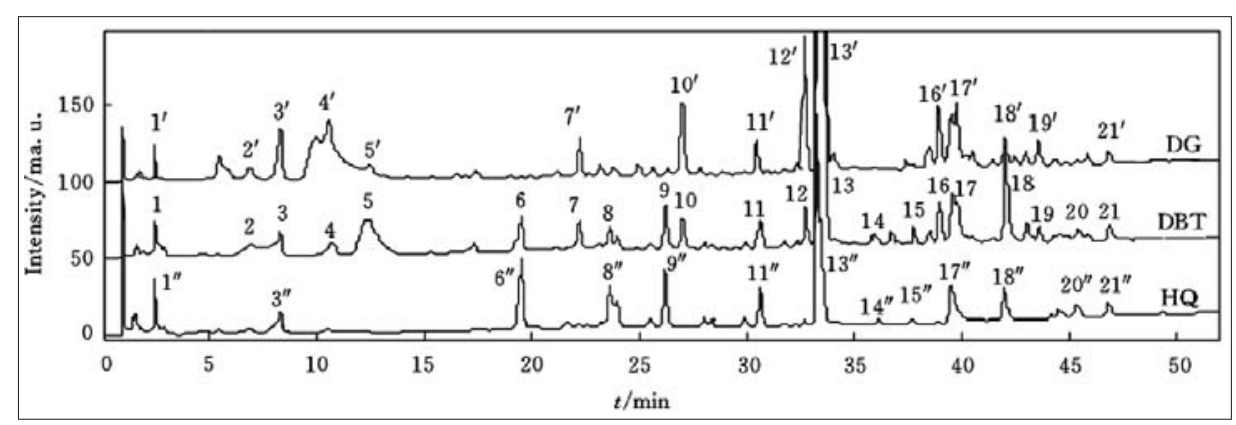

Fig. 4. Chromatographic fingerprints of Dangguibuxuetang (DBT), Danggui (DG (Radix Angelica sinensis)) and Huangqi (HQ, (Radix Astragali)) at the wavelength of $280 \mathrm{~nm}$. The peaks marked by numbers with and without primes indicate the same chemical compounds in $\mathrm{HQ}, \mathrm{DG}$ and DBT, respectively. Measurement conditions of chromatography: Hypersil ODS column: $415 \mu \mathrm{m}$, $416 \mathrm{~mm}$ i.d. $\times 250 \mathrm{~mm}$ (Bellefonte, PA, USA); mobile phase: $\mathrm{A}\left(\mathrm{H}_{2} \mathrm{O}\right)$ and $\mathrm{B}\left(\mathrm{CH}_{3} \mathrm{OH}\right)$; Graduated program: $1 \% \sim 25 \%$ of phase $B$ in phase $A$ during the period of $0 \sim 10 \mathrm{~min} ; 25 \% \sim 50 \%$ during the period of 10 20 $\mathrm{min} ; 50 \% \sim 70 \%$ during the period of 20 30 $\mathrm{min} ; 70 \% \sim 90 \%$ during the period of 30 50 min.

(Radix Angelica sinensis) and Huangqi (Radix Astragali) were compared for their relevant constituents (Fig. 4). It can be seen from the plot that of twenty-one strong peaks in Dangguibuxuetang, eight originate from Radix Angelica sinensis, six from Radix Astragali, and seven from both Radix Angelica sinensis and Radix Astragali. Also, some new chemical ingredients appeared in fingerprints of Dangguibuxuetang and some existing in the fingerprint of the single medicines contrarily disappeared after formulation. ${ }^{[43]}$ This seems to suggest that there might be certain interactions of the chemical ingredients in compound medicine in addition to the total sum effect of the single medicines.

Using the technique of chromatographic fingerprinting, the analysis of the products of roots of Semiaquilegia adoxoides at different locations was conducted. ${ }^{[25]}$ Also, the quality control for Cordyceps militaris with different genera was studied. ${ }^{[27]}$ With the help of GC-MS techniques, the chromatographic fingerprints of fresh and dry heartleaf houttuynia herbs were analyzed. The results showed that not only the fresh and dry heartleaf houttuynia herbs but also the different parts of the heartleaf houttuynia herbs could be successfully identified. ${ }^{29]}$ By combining HPLC and chemometric methods, such as principal component analysis (PCA) and partial least squares-linear discrimination analysis (PLS-LDA), Pericarpium Citri Reticulatae and Pericarpium Citri Reticulatae Viride could also be successfully pattern-analyzed and identified. [35]

TCM products are often made up of several herbs. The chromatographic fingerprints could also be used to address their quality stability and consistency. A simple and reproducible method based on HPLC was also developed to develop the chromatographic fingerprint of a complex herbal medicine, Shuang-Huang-Lian
(SHL) oral liquid. Ten batches of SHL obtained from different pharmaceutical factories were used to establish the fingerprint. [26]

In order to provide more information for complex TCM preparation, a twodimensional fingerprint (2D fingerprint) was established for various Qingkailing injections, which were produced by different manufacturers and procedures. [33] PCA of the 2D fingerprint data was performed in this study, and it led to an accurate classification of various samples with respect to their manufacturers and procedures. The quality of Qingkailing samples was further evaluated by similarity measures and the same results were achieved. ${ }^{[30,33]}$

\section{Simultaneously Qualitative and Quantitative analysis of TCM Fingerprints with the Help of Hyphenated Chromatographic Techniques and Chemometrics}

To understand bioactivities and and to enhance quality control of TCMs, it seems necessary to determine most of the phytochemical constituents of herbal products. With the development of analytical instruments, especially with the development of the hyphenated chromatographic instruments, such as GC-MS, HPLC-DAD, HPLC-DAD-MS ${ }^{\mathrm{n}}$, HPLC-NMR, CE-DAD, and CE-MS, the qualitative and quantitative ability has been enhanced significantly, since such instruments provide not only separation ability but also qualitative ability with spectroscopic profiles. This provides the chemical foundation for the explanation of the mechanism of pharmacological activity. Just as in metabolomics, as pointed out by Van de Greef, "the comprehensive identification of metabolites remains possible side effects of active compounds a key challenge".[44] Based on the CFs obtained, comprehensive identification of secondary metabolites of the TCMs should be very momentous, and the assistance of chemometric measurement is also indispensable.

In general, the data generated by hyphenated instruments are matrices with every row being a spectrum and every column a chromatogram at some wavelength, wave number or m/e unit. Commonly, the size of the data matrix obtained is rather large. However, the matrix data obtained by hyphenated chromatography has so-called dimension advantages as proposed by Booksh and Kowalski, ${ }^{[45]}$ which will make resolution of overlapping chromatographic peaks possible. Furthermore, the hyphenated technique might enhance the chromatographic separation ability by means of the additional spectral information, since one could easily find some useful component selectivity with the help of chemometric local rank analysis methods. ${ }^{[46]}$ Chromatography is a powerful tool for analyzing complicated systems such as TCM. However, as pointed out by Giddings, relative to the maximum peak content or peak capacity for closely spaced peaks, a random chromatogram will never contain more than about $37 \%$ of its potential peaks. Thus, the number of observed peaks is not the same as the number of distinct chemical components. ${ }^{[47]}$ On the other hand, a Chinese herbal formula for treating the common cold contains hundreds of chemical components, and it seems impossible to get a baseline separation with hundreds of analytes. As a result, identification of the purity of a target peak cluster followed by resolution into pure chromatograms and/or spectra is of great significance for complex systems like TCMs. Fortunately, the recently developed chemometrics resolution methods provide powerful tools to accomplish this job. ${ }^{[48-57]}$ With the help of chemometric resolution methods, the qualitative and relatively quantitative analysis for some CFs of volatile components in TCMs obtained from GC-MS were also reported. ${ }^{6,7,58-60]}$ When the boiling points of compounds are close to each other, co-elution of two or more different compounds is very possible although the chromatographic conditions are optimized. In such a case, by direct similarity searches in the MS database alone it is difficult to identify the compounds, which could even result in wrong conclusions. Therefore, it is extremely necessary to resolve the overlapping peaks by means of chemometric techniques. With the help of chemometric resolution methods, such as heuristic evolving latent projections and subwindow factor analysis, determination of volatile components in Cortex Cinnamomi, ${ }^{[6]}$ Notoptergium 
incium, ${ }^{[59]}$ Artemisia capillaris, ${ }^{[60]}$ and peptic powder ${ }^{[7]}$ were conducted by GCMS. Tentative identifications were performed by comparing the retention time and mass spectra of samples with standards or/and earlier publications.

Over the last decades, many chemometric techniques ${ }^{[61-72]}$ have been successfully applied to solve the problems arising from metabolic analysis, qualitative and quantitative analysis of herbal medicines (HM), and others. ${ }^{[65-70]}$ In addition the SIM technique was developed for the analysis of complex chromatographic systems. ${ }^{[71,72]}$ But the identification and quantification of all chemical components in a complex system is far from satisfactory. [73]

Fortunately, in some cases, it is not necessary to resolve all the ingredients in complex systems, but only to identify parts of them or find the difference and similarities of components between two datasets of target samples for purpose of quality control of TCMs. It is obvious that the integrated investigation must be time-saving and effective for obtaining the valuable information. In the relationship between the two chromatographic fingerprints (CF), five possible relationships of the chemical components between two different and complex systems are illustrated in Fig. 5. To analyze the five possible relationships, a novel chemometric method was developed. The method, named alternative moving window factor analysis (AMWFA), could utilize the cross information hidden in the two systems to determine the number of common components between them and even to identify their corresponding spectra semi-automatically. The selective information in matrices $\mathbf{X}$ and/or $\mathbf{Y}$ can alternatively be employed for the identification of their common components. Using component information in one system to deduce the spectrum in the other related system is the main advantage of AMWFA, since the same component should have the same spectrum even if they exist in different fingerprints. When no or only weak selective information exists in them, the strategy of sub-matrices extraction from data $\mathbf{X}$ and/ or $\mathbf{Y}$ can be used for identification with the moving window technique. The working procedure of AMWFA is illustrated in Fig. 6 . From the results of comparative analysis of volatile chemical components in 'TCM drugs pair' Rhizoma ligustici chuanxiongRadix paeoniae rubra and the involved two single HMs and Angelica oral solution and its plasma sample after oral intake by rabbit, the powerful ability of the method is shown. ${ }^{[74]}$ Also, with the help of AMWFA, the volatile components between stems and roots and also among five Clematis species from China were studied and analyzed by GC-MS. Tentative identification of the compounds was assisted by compar-
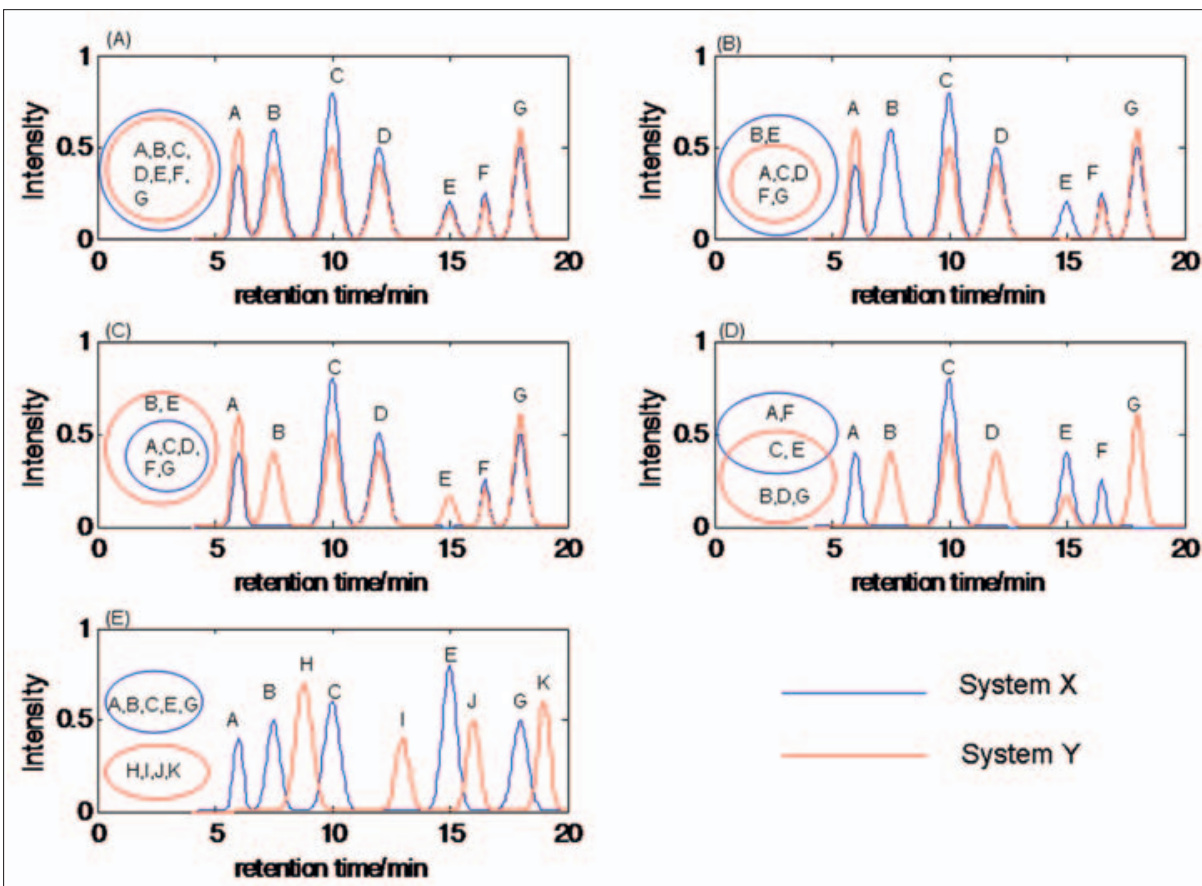

System X

System $Y$

Fig. 5. Schematic illustration of the five possible relationships of chemical components contained in two different complex chromatographic systems $\mathbf{X}$ and $\mathbf{Y}$. The overstriking maculae in $\mathbf{X}$ and $\mathbf{Y}$ denote different components.

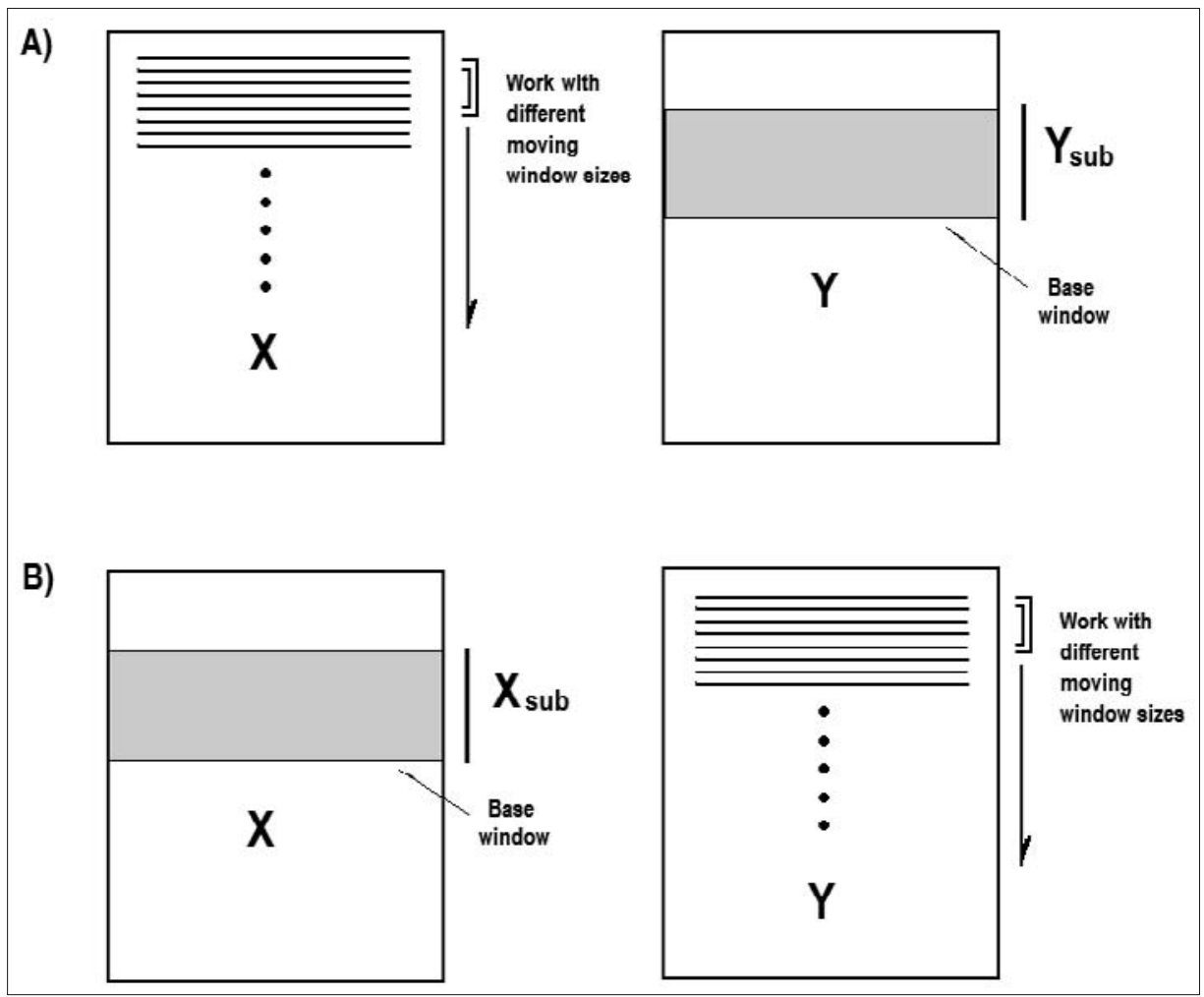

Fig. 6. Illustration of working procedure of AMWFA for defining the target and base matrices. Top part: $\mathbf{X}$ as the target matrix and $\mathbf{Y}$ as the base matrix; bottom part: $\mathbf{Y}$ as the target matrix and $\mathbf{X}$ as the base matrix alternatively.

ison of temperature-programmed retention indices with authentic samples. ${ }^{[75]}$

In addition, the similarities and differences of essential oil components in pCRV and pCR were investigated by GCMS combined with the help of AMWFA. The essential oils from pCRV and pCR differed significantly both qualitatively and quantitatively. The main compound in the essential oils from pCRV and pCR was D-limonene accounting for $65-83 \%$. Essential oils from four samples of Rhododendron were analyzed by GC-MS. A total of 128 volatile components were identified temporarily with the help of retention indices and the chemometrics 
resolution method.[76] A compound medicine of Dangguibuxuetang and its two involved single medicines of Danggui (Radix Angelica sinensis) and Huangqi (Radix Astragali) were compared for their relevant constituents. It was found that of twenty-one strong peaks in Dangguibuxuetang, eight originate from Radix Angelica sinensis, six from Radix Astragali, and seven from both Radix Angelica sinensis and Radix Astragali. Some new chemical ingredients appeared in fingerprints of Dangguibuxuetang, and some existing in those of single medicines contrarily disappeared after formulation. It is suggested that there might be certain interactions of the chemical ingredients in compound medicine besides their total sum effect of single medicines. ${ }^{[77]}$

It is worth noting that the comparison analysis of CFs could be used to trace the metabolic situation of TCMs. From the point of view of exploring the mechanism of why TCM works, this kind of research might be of great importance. If one could trace the metabolic changes of the TCMs when they are taken into the body of an animal/human being, it would be very helpful to screen active components and/or the pharmacokinetics. Based on the metabolic fingerprinting technique and LC/DAD-MS, a rapid screening and analysis of the multiple absorbed bioactive components and metabolites of an oral solution of Danggui and the Dangguibuxue decoction (DD) in rabbit plasma after oral administration were developed. [78-80] The results obtained from a comprehensive comparative analysis of the fingerprints of the DD and its metabolic fingerprints in rabbit plasma indicated that 46 components in the DD were absorbed into the rabbit's body. Of them, ten components were tentatively identified from their MS and UV spectra and retention behaviors by comparing the results with the reported literature were assessed. In addition, 21 components were found in the metabolic fingerprints only, which suggested that they might be metabolites of some components in the DD. This might be, in our opinion, important not only for the pharmaceutical discovery process and the quality control of crude drugs, but also could be a helpful aid in discovering the curative mechanism of TCMs.

\section{Exploration of the Relationship between Bioactivity and Composition of TCM and Validation of their Bioactive Modes with the Help of Chemometrics}

With the chemical composition of TCMs at hand, the exploration of the relationship between the CFs and efficacy of the HMs seems thus to be the important aspect for the quality control of HMs. However, evaluating reasonably their relationship is obviously not a trivial task. Recently, some work has been done along this direction with the help of chemometrics. ${ }^{8}{ }^{81-86]}$

The quantitative relationship between chemical composition and cholesterollowering effect of Qi-Xue-Bing-ZhiFang, a widely used HM in China, was investigated. Quantitative compositionactivity relationship models generated by multiple linear regression, artificial neural networks, and support vector regression exhibited different capabilities of predictive accuracy. Moreover, the proportion of two active components of Qi-Xue-Bing-Zhi-Fang was optimized based on the quantitative compositionactivity relationship model to obtain a new formulation. Validation experiments showed that the optimized HM has greater activity. ${ }^{[85]}$

Many herbal products are claimed to have healing or protective effects, though often these activities are neither evaluated nor quantified. For instance, it was shown that green tea exhibits protective effects against several cancers introduced by chemical carcinogens as well as against atherosclerosis and coronary heart diseases. ${ }^{[86]}$ These positive actions of green tea are related to its antioxidant capacity and therefore an attempt was made to predict the total antioxidant capacity of green tea from CFs by linear multivariate calibration techniques.[87] The predicted antioxidant capacities can then be a measure for the protective effects and for the quality of the tea. However, for chromatographic data, e.g. fingerprints, only a few multivariate calibration applications are described. Recently, Dumarey et al. explored linear multivariate calibration techniques to predict the total antioxidant capacity of green tea from CFs. Also, van Nederkassel et al. ${ }^{[83]}$ predicted the total antioxidant capacity of green tea from their CFs by the use of PLS ${ }^{[87,88]}$ and uninformative variable elimination PLS.[89]

More recently, using whole chromatographic profiles and measurements of total bioactivity as input, a quantitative patternactivity relationship approach ${ }^{[90]}$ was proposed as a general method for providing two pieces of crucial information about complex bioactive mixtures available: i) a model for predicting total bioactivity from the $\mathrm{CF}$ and ii) the features in the chromatographic profile responsible for the bioactivity. The targeted approach makes information about bioactivity available at the molecular level and provides possibilities for assessment of HMs possible beyond just authentication and total bioactivity. As an example, the antioxidant property of the HM Radix Puerariae lobatae was measured for its reducing power toward a ferric ion complex. A PLS model was created to predict the antioxidant activity from the CF. Using the antioxidant activity as a target, the most discriminatory projection in the multivariate space spanned by the chromatographic profiles was revealed.

On the other hand, the mechanism of action of TCMs is usually unknown, which also poses challenges to the pharmaceutical and agrochemical industries. ${ }^{\text {91-93] }}$ The metabolic profile is a very sensitive indicator of environmental influences and might be used to detect and analyze changes of the total metabolic state of microbes due to pathophysiological stimuli, ${ }^{[94]}$ which makes it an attractive candidate for mode of action studies. ${ }^{[95]}$ Metabolic profiling is being used to evaluate the pharmacological mechanism of the drugs or drug candidates. The method is particularly useful when a moderate number of different outcomes (e.g. modes-of-action, disease states) can be pre-defined. ${ }^{[96]}$

Using the metabolomic profile and nine antibacterial substances with known modes of action, the antibacterial mode of berberine on Staphylococcus aureus was recently investigated. ${ }^{[97]}$ The working procedure is elucidated briefly in Fig. 7. With the help of HPLC/ESI-MS, metabolic profiles of $S$. aureus treated by berberine and nine antibacterial substances with known modes of action (see Table 1) were first acquired. After data pretreatment, the acquired profiles were reduced into several MS vectors containing $900 \mathrm{~m} / \mathrm{z}$ values. Then, PCA was carried out upon those metabolic profiles in order to classify those drugs according to their mechanisms. From the result obtained by PCA, the possible antibacterial mode of berberine was explored. Also the antimicrobial roles of dihydrocucurbitacin F-25-O-acetate, one of the major components in Hemsleya pengxianensis, on $S$. aureus were also explored with the similar approach. ${ }^{[98]}$ The result obtained showed the mechanism may be to inhibit cell wall synthesis. Another example of score plot from PCA (the variance of the first two PC being $89.5 \%$ of total variance of the data) for exploring the antibacterial mode of TCM Aquilegia oxysepala is shown in Fig. 8. From Fig. 8, one can see clearly that 90 samples treated with different drugs and controls are wellseparated. Cefataxime, whose target is on transpeptidases and carboxpeptidases, formed a distinct cluster separate from the other antibiotics based on its different mode of action. Acheomycin, lincolmensin, erythromycin, chloromycetin, and streptomycin cluster together. As known from Table 1, lincolmensin, erythromycin, and chloromycetin have effects on the 


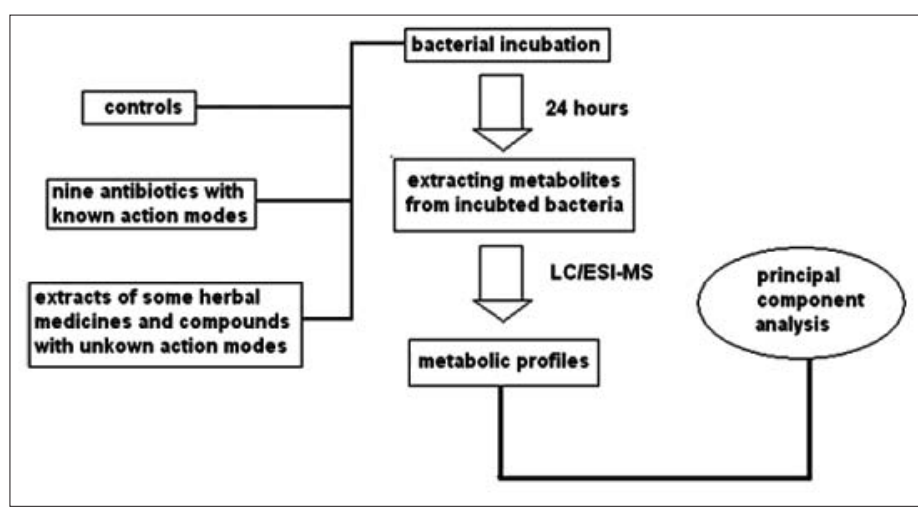

Fig. 7. Working

procedure of validation of antibacterial mode of traditional Chinese medicine by metabolomics and PCA.

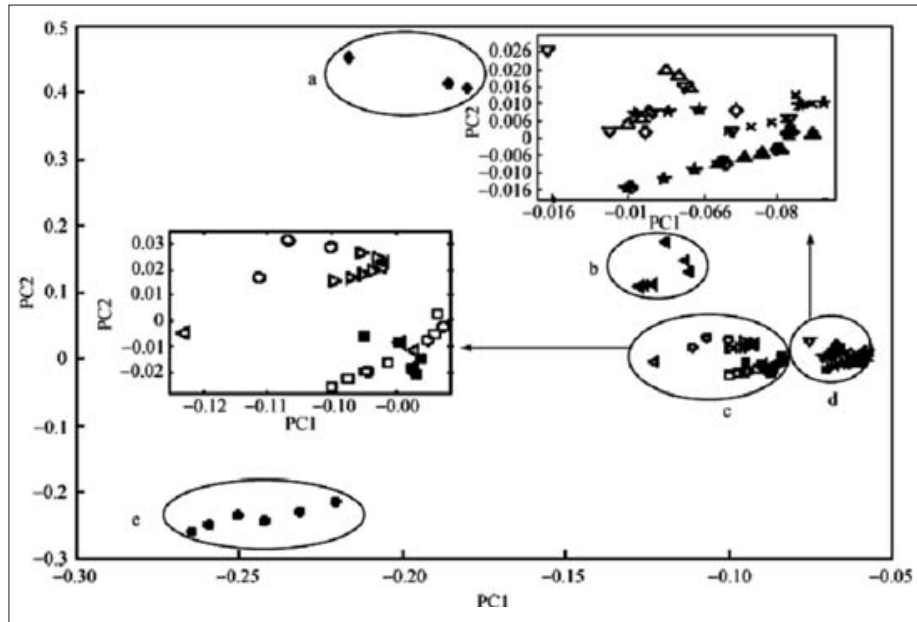

Fig. 8. PCA projection of metabolic profiles of controls and cultures treated with Aquilegia oxysepala, genkwanin, apigenin, maguoflorine, berberine, and nine antibiotics. Controls $(\diamond)$, acheomycin $(\triangle)$, lincolmensin $(x)$, erythromycin $(\mathbf{\Lambda})$, chloromycetin (iz), streptomycin ( $\star$ ), cefataxime ( $\triangleleft)$, vancomycin (๑), rifampicin ( $\square$ ) norfloxacin ( $\square$ ), A. oxysepala $(\diamond)$, genkwanin $(\bigcirc)$, maguoflorine $(\nabla)$, apigenin $(\triangleright)$, and berberine $(\triangleleft)$.

\section{Conclusion}

The parameters relevant to the quality of Chinese herbal medicines acquired from chromatographic fingerprinting certainly form a large dataset, which can only be processed sufficiently and systematically through chemometric calculation. The integrated quality information can be explored thoroughly as a whole to extract logical conclusions from the empirical evidence, particularly when the compound data derived from a macro amount of samples are subjected to a series analysis by means of chromatography hyphenated with various detective measurements. Furthermore, the quality information serves as a "quality databank' in order to incorporate phytochemical information with biological activity of the herbals so as to seek the relationship between the $\mathrm{CF}$ and the synergic efficacy of the TCMs. ${ }^{[109-111]}$ Basically, chemical fingerprint analysis aided by chemometrics can ensure the maintenance of the consistent quality of samples for biological and pharmacological research.

\section{Acknowledgement}

This work is financially supported by the National Nature Foundation Committee of P. R. China (Grant No. 20875104) and the international cooperation project on traditional Chinese medicines of Ministry of Science and Technology of China (2007DFA40680).

Received: October 9, 2011

[1] L. Zhao, K. Chan, Chin. J. Integr. Med. 2003, 9, 299.

[2] W. Y. Jiang, Trends in Pharmacol. Sci. 2005, 26 , 558.

[3] World Health Organization (WHO), WPR/ RC52/7: 'A Draft Regional Strategy for Traditional Medicine in Western Pacific', WHO Regional Committee, 52nd Session Brunei Darussalam, 2001, 9, 10.

[4] O. Hiroko, Y. Sukeo, K. Tetsuo, H. Shigeyoshi, M. Toshiaki, K. S. Ryan, M. Takeyuki, K. Kenzo, T. Toshiro, Cancer 1995, 76, 743.

[5] M. P. Sheehan, D. J. Atherton, Br. J. Dermatol. 1992, 126, 179 .

[6] M. P. Sheehan, M. H. A. Rustin, C. Buckley, D. J. Harris, L. Ostlere, A. Dawson, D. J. Atherton, J. Brostoff, 1992, 340, 13.

[7] R. R. Chaudhury, 'Herbal Medicine for Human Health', WHO regional publication, SEARO, no. 20, WHO, New Delhi, India, 1992, pp. 2627.

[8] K. Chan, Trends Pharmacol. Sci. 1995, 16, 182.

[9] T. Xue, R. Roy, Science 2003, 300, 740.

[10] M. G. Lee, C. Wynder, N. Cooch, R. Shiekhattar, Nature 2005, 437, 432

[11] S. Mencher, L. Wang, BMC Clin. Pharmacol. $\mathbf{2 0 0 5}, 5,3$.

[12] H. Kitano, Nat. Rev. Drug Discov. 2007, 6, 202.

[13] Y. Z. Liang, P. S. Xie, K. Chan, J. Chromatogr. B. Analyt. Technol. Biomed. Life Sci. 2004, 812, 53.

[14] M. Ganzera, Plant Medica 2009, 75, 776.

[15] S. Liu, B.Wang, X. Z. Li, L. F. Qi, Y. Z. Liang, J. Sep. Sci. 2009, 32, 2476.

[16] State Drug Administration of China, 'Technical Requirements of the Fingerprint in Injection of
Cefataxime Peptidoglycan synthesis

Vancomycin Peptidoglycan synthesis
Transpeptidases and carboxypeptidases Cell wall peptidoglycan 
Chinese Materia Medica (Tentative Standard)', Chin. Tradit. Pat. Med. 2000, 22, 671.

[17] E. S. Ong, J Sep Sci. 2002, 25, 825.

[18] P. S. Xie, Tradit. Chin. Drug Res. Clin. Pharm. 2001, 12, 141 .

[19] G. H. Lu, K. Chan, Y. Z. Liang, K. Leung, C. L. Chan, Z. H. Jiang, Z. Z. Zhao, J. Chromatogr. A 2005, 1073, 383 .

[20] P. S. Xie, S. B. Chen, Y. Z. Liang, X. H. Wang, R. T. Tian, R. Upton, J. Chromatogr. A 2006, 1112,171

[21] Y. N. Ni, L. S. Zhang, J. Churchill, S. Kokot, Talanta 2007, 72, 1533.

[22] P. Zou, Y. Hong, H. L. Koh, J. Pharmaceut Biomed. 2005, 38, 514

[23] Y. Xie, Z. H. Jiang, H. Zhou, X. Cai, Y. F. Wong, Z. Q. Liu, Z. X. Bian, H. X. Xu, L. Liu, J. Pharmaceut. Biomed. 2007, 43, 204.

[24] A. M. van Nederkassel, V. Vijverman, D. L. Massart, Y. V. Heyden, J. Chromatogr. A $\mathbf{2 0 0 5}$ 1085, 230

[25] F. Niu, Z. Niu, G. Xie, F. Meng, G. Zhang, Z Cui, P. F. Tu. Chromatographia 2006, 64, 593.

[26] R. M. Yu, B. Ye, C. Y. Yan, L. Y. Song, Z. Zhang, W. Yang, Y. Zhao, J. Pharmaceut. Biomed. 2007, 44, 818

[27] C. Chen, H. Zhang, W. Xiao, Z. P. Yong, N. Bai, J. Chromatogr. A 2007, 1154, 250.

[28] Y. H. Cao, L. C. Wang, X. J. Yu, J. N.Ye, J. Pharmaceut. Biomed. Anal. 2006, 41, 845

[29] J. Meng, K. S. Y. Leung, Z. H. Jiang, X. P. Dong, Z. Z. Zhao, Chem. Pharm. Bull. 2005 , $53,1484$.

[30] S. K. Yan, W. F. Xin, G. A. Luo, Y. M. Wang, Y. Y. Cheng, J. Chromatogr. A 2005, 1090, 90.

[31] Z. D. Zeng, Y. Z. Liang, F. T. Chau, Y. L. Wang, Anal. Bioanal. Chem. 2006, 385, 392.

[32] A. J. Lau, B. H. Seo, S. O. Woo, H. L. Koh, J. Chromatogr. A 2004, 1057, 141.

[33] X. H. Fan, Y. Y. Cheng, Z. L. Ye, R. C. Lin, Z. Z Qian, Anal. Chim. Acta 2006, 555, 217.

[34] Y. G. Li, F. Zhang, Z. T. Wang, Z. B. Hu, J. Pharmaceut. Biomed. 2004, 35, 1101.

[35] L. Z. Yi, D. L. Yuan, Y. Z. Liang, P. S. Xie, Y. Zhao, Anal. Chim. Acta 2007, 588, 207.

[36] P. K. Li, B. L. Guo, W. H. Huang, Chin. J. Chin. Mater. Med. 2008, 1662.

[37] B. L. Guo, L. K. Pei, P. G. Xiao, J. Sys. Evol. 2008, 46, 874.

[38] C. Y. Huang, L. H. Zhao, L. H. Mei, J. Nat. Med. 2003, 1, 146 .

[39] L. X. Wang, C. Z. Wang, X. D. Geng, Acta Chim. Sin. 2006, 64, 551.

[40] W. T. Stearn, J. Linn. Soc. 1938, 51, 409.

[41] W. T. Stearn, 'The genus Epimedium and other herbaceous Berberidaceae including the genus Podophyllium', Oregon, Timber Press, 2002, pp 35-36.

[42] P. S. Xie, Y. Z. Yan, B. L. Guo, C. W. K. Lam, S H. Chui, Q. X. Yu, C, J. Pharm. Biomed. Anal. 2010, 52, 452 .

[43] Y. L. Wang, Y. Z. Liang, Y. Hu, B. Y. Li, Z. D. Zeng, S. P. Xu, Chem. J. Chinese Univ. 2006, 27, 222.

[44] J. Van der Greef, P. Stroobant, R. van der Heijden, Curr. Opin. Chem. Biol. 2004, 8, 559.

[45] K. S. Booksh, B. R. Kowalski, Anal. Chem. 1994, 66, 782

[46] F. Gong, Y. Z. Liang, Q. S. Xu, Anal. Chim. Acta 2001, 450, 99.

[47] J. M. Davis, J. C. Giddings, Anal. Chem. 1983, $55,418$.

[48] W. E. Lawton, E. A. Sylvestre, Technometrics 1971, 13, 617.
[49] O. M. Kvalheim, Y. Z. Liang, Anal. Chem. 1992, 64, 936

[50] Y. Z. Liang, O. M. Kvalheim, H. R. Keller, D. L. Massart, Anal. Chem. 1992, 64, 946.

[51] E. R. Malinowski, 'Factor Analysis in Chemistry', 2nd ed., Wiley, New York, 1991

[52] K. Faber, B. R. Kowalski, J. Chemom. 1997, 11, 53.

[53] J. Mandel, Technometrics 1978, 20, 397.

[54] R. F. Hirsch, G. L. Wu, P. C. Tway, Chemom. Intell. Lab. Syst. 1987, 1, 265.

[55] Y. Z. Liang, O. M. Kvalheim, A. Hoskuldsson, Chemo. Intelli. Lab. Sys. 1993, 7, 277.

[56] R. Boque, F. X. Rius, Chemom. Intell. Lab. Syst. 1996, 32, 11

[57] Y. Z. Liang, L. Z. Yi, Q. S. Xu, Science in China Series B-Chemistry 2008, 51, 718.

[58] F. Gong, Y. S. Fung, Y. Z. Liang, J. Agri. Food Chem. 2004, 52, 6378.

[59] F. Q. Guo, Y. Z. Liang, C. J. Xu, X. N. Li, L. F. Huang, J. Pharmaceut. Biomed. 2004, 35, 469.

[60] B. Y. Li, Y. Hu, Y. Z. Liang, L. F. Huang, C. J. Xu, P. S. Xie, J Sep. Sci. 2004, 27, 581.

[61] R. Tauler, E. Casassas, Chemom. Intell. Lab. Syst. 1992, 14, 1.

[62] R. Tauler, Chemom. Intell. Lab. Syst. 1995, 30 , 133.

[63] R. Tauler, D.Barcelo, Trends Anal. Chem. 1993, 12, 319 .

[64] R. Tauler, S. Lacorte, D. Barcelo, J. Chromatogr. 1996, 730, 177

[65] M. Maeder, Anal. Chem. 1987, 59, 527.

[66] M. Maeder, A. Zilian, Chemom. Intell. Lab. Syst. 1988, 3, 205.

[67] E. R. Malinowski, J. Chemom. 1992, 6, 29.

[68] E. R. Malinowski, J. Chemom. 1996, 10, 273.

[69] Y. Z. Liang, O. M. Kvalheim, A. Rahmani, R. Brereton, J. Chemom. 1993, 7, 15.

[70] R. Manne, H. L. Shen, Y. Z. Liang, Chemom. Intell. Lab. Syst. 1999, 15, 171.

[71] W. Bashir, B. Paull, J. Chromatogr. A 2001, 910, 301.

[72] H. Z. Wan, K. Stacey, F. Johnand, J. Cacia, J. Chromatogr. A 2001, 913, 437.

[73] M. T. Lohnes, R. D. Guy, P. D. Wentzell, Anal. Chim. Acta 1999, 389, 95

[74] Z. D. Zeng, Y. Z. Liang, Y. L. Wang, X. R. Li, L. M. Liang, Q. S. Xu, C. X. Zhao, B. Y. Li, F. T. Chau, J. Chromatogr. A 2006, 1107, 273.

[75] Y. X. Zeng, C. X. Zhao, Y. Z. Liang, H. Yang, H. Z. Fang, L. Z. Yi, Z. D. Zeng, Anal. Chim. Acta 2007, 595, 328

[76] Y. M. Wang, L. Z. Yi, Y. Z. Liang, H. D. Li, D. L. Yuan, H. Y. Gao, M. M. Zeng, J. Pharm. Biomed. 2008, 46, 66

[77] Y. L. Wang, Y. Z. Liang, Y. Hu, B.Y. Li, Z. D. Zeng, S. P. Xu, Chem. J. Chinese Univ. 2006, 27, 222.

[78] Y. L. Wang, Y. Z. Liang, Y. Hu, B.Y. Li, Z. D. Zeng, Y. K. He, Chemom. Intell. Lab. Syst. 2006, 82, 229.

[79] Y. L. Wang, Y. Z. Liang, B. M. Chen, Y. K. He, B.Y. Li, Q. N. Hu, Anal. Bioanal. Chem. 2005, 383, 247.

[80] P. Wang, Y. Z. Liang, N. Zhou, B. M. Chen, L. Z. Yi, Y. Yu, Z. B. Yi, Rapid Commun. Mass Sp. 2007, 21, 99 .

[81] M. Dumarey, A. M. van Nederkassel, E. Deconinck, Y. Vander Heyden, J. Chromatogr. A 2008, 1192, 81.

[82] M. Daszykowski, Y. Vander Heyden, B. Walczak, J. Chromatogr. A 2007, 1176, 12.

[83] A. M. Van Nederkassel, M. Daszykowski, D. L. Massart, Y. Vander Heyden, J. Chromatogr. A 2005, 1096, 177.
[84] Y. Y. Cheng, Y. Wang, X. W. Wang, Comput. Biol. Chem. 2006, 30, 148.

[85] Y. Wang, X. W. Wang, Y. Y. Cheng, Chem. Biol. Drug Des. 2006, 68, 166.

[86] H. Mukhtar, N. Ahmad, Am. J. Clin. Nutr. 2000, $71,1698 \mathrm{~S}$

[87] B. G. M. Vandeginste, D. L. Massart, L. M. C. Buydens, S. De Jong, P. J. Lewi, J. SmeyersVerbeke, 'Handbook of Chemometrics and Qualimetrics: Part B', Elsevier, Amsterdam, 1998.

[88] D. L. Massart, B. G. M. Vandeginste, L. M. C. Buydens, S. De Jong, P. J. Lewi, J. SmeyersVerbeke, 'Handbook of Chemometrics and Qualimetrics: Part A', Elsevier, Amsterdam, 1997.

[89] V. Centner, D. L. Massart, O. E. deNoord, S. de Jong, B. G. M. Vandeginste, C. Sterna, Anal. Chem. 1996, 68, 3851.

[90] F. T. Chau, H. Y. Chan, C. Y. Cheung, C. J. Xu, Y. Z. Liang, O. M. Kvalheim, Anal. Chem. 2009, 81, 7217.

[91] A. J. Corran, A. Renwick, S. Dunbar, Pestic. Sci. 1998, 54, 338 .

[92] F. Spaltmann, M. Blunck, K. Ziegelbauer, Drug Discov. Today 1999, 4, 17.

[93] T. A. White, D. B. Kell, Curr. Opin. Biotechnol. 2004, 15, 304.

[94] J. Lindon, Business Briefing: Future Drug Discovery 2004, 1-6.

[95] M. L. Anthony, B. C. Sweatman, C. R. Beddell, Mol. Pharmacol. 1994, 46, 199.

[96] E. D. Brown, G. D. Wright, Chem. Rev. 2005, 105, 759.

[97] Z. B. Yi, Y. Yu, Y. Z. Liang, B. Zeng, J. Pharm. Biomed. 2007, 44, 301

[98] Z. B. Yi, Y. Yu, Y. Z. Liang, J. Ethnopharmacol. 2008, 116, 89

[99] C. L. Kuo, C. C. Chou, Cancer Lett. 1995, 93 , 193.

[100] W. Y. Li, H. Lu, C. X. Xu, J. B. Zhang, Z. H Lu, Spectrosc. Lett. 1998, 31, 1287.

[101] S. Mazzini, M. C. Bellucci, R. Mondelli, Bioorg. Med. Chem. 2003, 11, 505.

[102] R. C. Yadav, G. S. Kuma, K. Bhadra, P. Giri, R. Sinha, S. Pal, M. Maiti, Bioorg. Med. Chem. 2005, 13, 165.

[103] W. H. Chen, C. L. Chan, Z. Cai, G. A. Luo, Z H. Jiang, Bioorg. Med. Chem. Lett. 2004, 14, 4955.

[104] W. H. Chen, J. Y. Pang, Y. Qin, Q. Peng, Z. W. Cai, Z. H. Jiang, Bioorg. Med. Chem. Lett. 2005, 15, 2689.

[105] D. Debnath, G. S. Kumar, M. J. Mait, Biomol. Struct. Dyn. 1991, 9, 61

[106] Y. Yu, Z. B. Yi, Y. Z. Liang, Bioorg. Med. Chem. Lett. 2007, 17, 1855.

[107] Y.Yu, Z. B. Yi, Y. Z. Liang, FEBS Lett. 2007, $581,4179$.

[108] Z. B. Yi, Y. Yu, Y. Z. Liang, B. Zeng, LWT 2008, 41, 597.

[109] X. J. Yan, J. J. Zhou, G. R. Xie, 'Traditional Chinese Medicines: Molecular Structures, Natural Sources and Applications', Aldershot, Ashgate, 1999

[110] P. H. Raven, R. F. Evert, S. E. Eichhorn, 'Biology of Plants', 6th Edn, Freeman, New York 1999

[111] R. Bauer, Drug Inform. J. 1998, 32, 101; Y Liang, P. Xie, F. Chau, J. Sep. Sci. 2010, 33, 410 . 